\title{
COVID 19 AND DIABETES
}

\section{E. PRABHAKAR REDDY ${ }^{1 *}$, T.MOHANALAKSHMI ${ }^{2}$ AND C. NAVEEN KUMAR ${ }^{3}$}

1: Professor in Biochemistry, Bharath Medical College and Hospital, Chennai, Affiliated to BIHER

2: Associate Professor in Microbiology, Sri Lakshmi Narayana Institute of Medical Sciences, Puducherry, Affiliated to BIHER

3: Associate Professor in Microbiology, Sri Lakshmi Narayana Institute of Medical Sciences, Puducherry, Affiliated to BIHER

*Corresponding Author: Dr. E. Prabhakar Reddy: E Mail: drpebyreddy@yahoo.com Received 17 $7^{\text {th }}$ Oct. 2020; Revised $16^{\text {th }}$ Nov. 2020; Accepted $12^{\text {th }}$ Dec. 2020; Available online $1^{\text {st }}$ Jan. 2021 https://doi.org/10.31032/IJBPAS/2021/10.1.1011

ABSTRACT

Older people and people with pre-existing medical conditions (such as diabetes, heart disease and asthma) appear to be more vulnerable to becoming severely ill with the COVID19 virus. When people with diabetes develop a viral infection, it can be harder to treat due to fluctuations in blood glucose levels and, possibly, the presence of diabetes complications. patients with COVID-19 with diabetes have a worse prognosis, most probably because of the concurring effect of multiple factors. The prevalence of diabetes increases with age in both the general population and in patients with COVID-19. Diabetic patients should keep their blood sugar under control by doing frequent monitoring using digital appliance and to contact with their physician to remain in good glycemic control. Future research is urgently needed to provide a better understanding regarding potential differences in genetic predispositions across populations, underlying pathophysiological mechanisms of the association between COVID-19 and diabetes, and its clinical management.

Keywords: Corona virus, Hyperglycemia, Diabetes mellitus, Diet and exercise 


\section{INTRODUCTION}

The prevalence of diabetes for all age groups worldwide was estimated to be $2.8 \%$ in 2000 and $4.4 \%$ in 2030 . The total number of people with diabetes is projected to rise from 171 million in 2000 to 366 million in 2030 [1]. Diabetes mellitus is a group of metabolic disorders of carbohydrate characterized by hyperglycemia. Hyperglycemia of diabetes mellitus (DM) generates reactive oxygen species (ROS) which causes oxidative damage to lipids. Diabetes is associated with various micro vascular and macro vascular complications. This oxidative stress of diabetes is found to be associated with a depleted natural anti oxidant reserve. The prevalence of diabetes is rapidly rising all over the globe at an alarming rate. According to the International Diabetes Foundation (IDF), the total number of diabetic subjects is to be around 40.9 million in India at present which is expected to rise to 69.9 million by the year 2025 [2].

People of all ages can be infected. For many (more than $80 \%$ of cases), COVID-19 is mild, with minimal flu-like symptoms. Some have not shown symptoms or only very mild symptoms, more like a common cold. The majority of people who have caught the virus have not needed to be hospitalised for supportive care. However, in up to $15 \%$ of cases COVID-19 has been severe and in around $5 \%$ of cases it has led to critical illness. The vast majority (around 98\%) of people infected to date have survived. Older people and people with pre-existing medical conditions (such as diabetes, heart disease and asthma) appear to be more vulnerable to becoming severely ill with the COVID-19 virus. When people with diabetes develop a viral infection, it can be harder to treat due to fluctuations in blood glucose levels and, possibly, the presence of diabetes complications. There appear to be two reasons for this. Firstly, the immune system is compromised, making it harder to fight the virus and likely leading to a longer recovery period. Secondly, the virus may thrive in an environment of elevated blood glucose.

\section{DISCUSSION}

Now the Fastest culture in the cities develops and moves from traditional to modern living life style in the diet and physical activity. Diet and exercise are important components of the treatment strategy for adults with type 2 diabetes. Both are important factors for treatment to adults with type 2 Diabetes and it improves the insulin sensitivity and glycemic control and it decreases the medications and insulin. Faulty diet makes the best of 
medicine in effective. Based on our study we focused on dietary habits and excercise behaviour of type 2 diabetes. Causes of increase in incidence of diabetics include, improved nutrition, better hygiene, control of many communicable disease. It improve access to quality healthcare and the main drivers of diabetes epidemic in India are fast food culture and sedentarinism.

Many foreigners were wondered that South Indians have great respect for the therapeutic value of food as followed in Ayurvedic principles in their everyday eating like served food on a banana leaf, daily intake of Pepper, Tamarid and turmeric powder added as an ingredients in most of the recipe in the South Indian dishes like Pongal, Rasam and Sambar recipes which showed antiviral property by inhibiting the viral replication [3-5]. To give information to the diabetics to not to take foods like rich in carbohydrate, fat and other fleshy foods. $50 \%$ of the people were had family history of diabetes and most of them were in allopathic treatment. They were well known of the foods to be restricted hypoglycemic foods and other special foods beneficial for diabetes progression of the management. $60 \%$ of the people were exercise mostly in the form of walking and formers are working regularly in the fields. As eating healthy foods not only regulate the immune system but also protect against infection. Further Ginger and garlic added in most of the South Indian recipes also reported to have antiviral property by increase the body temperature. An increase in body temperature has been known since ancient times to be associated with infection and inflammation. Elevated body temperature stimulate more number of CD8 or cytotoxic T-cell" which capable of destroying the intracellular parasite [6]. In the present study $86 \%$ of studied population had ancient style of traditional food. Traditionally $88 \%$ of population use to light oil lamp and $62 \%$ of population use Benzoin resin smoke after Sun set which will act as an air purifier, remove moisture content and act as organic disinfectants that drive away insects and many pathogenic microbes of airborne [7].

Poor hygiene practices and inadequate sanitary conditions result in wide spread of communicable diseases. In the present awareness of hand washing was assessed in which $94 \%$ of people are washing hands with soap before taking food and only $62 \%$ of people were using Handkerchief while coughing and sneezing warrant further sensitize. South India Greeting culture is by clasp vertically hands in front of the chest without any physical contacts and strictly restricted the footwear's away from the living area also 
plays an important role in control the transmission of contagious infection.

In a British cohort of 5693 patients with COVID-19 in hospital, the risk of death was more common in those with uncontrolled diabetes (hazard ratio [HR] 2.36, 95\% CI 2.18-2.56) [8]. A survey done in England (UK) [9] showed that of 23804 patients with COVID-19 dying in hospital, $32 \%$ had type 2 diabetes and $1 \cdot 5 \%$ had type 1 diabetes, with 2.03 and 3.5 times the odds of dying compared with patients without diabetes, respectively.

Patients with COVID-19 with diabetes have a worse prognosis, most probably because of the concurring effect of multiple factors. The prevalence of diabetes increases with age in both the general population and in patients with COVID-19. Accordingly, the average age of patients with COVID-19 with diabetes is older than those without diabetes. Advanced age and comorbidities, such as diabetes mellitus, high blood pressure, and history of cerebrovascular accidents are reported to have worse outcome. Chronic inflammation by cytokine storm and direct insult to pancreatic by COVID-19 might be postulated mechanisms of inducing or deteriorating diabetes. Individualized patient-centric treatment and optimal blood sugar control should be made based on disease severity, presence of comorbid condition, and complications related to diabetes, age, and other risk factors [10]. Recent clinical trials have shown some hope to anti-interleukin antibody as a potential therapeutic option against COVID-19 especially in people with severe illness.T2 DM with severe COVID-19 infection are more prone for a cytokine storm characterized by increased titer of inflammatory cytokines IL-2R and IL-6 and chemokines. [11]. ARDS, an immunopathological changes in lungs and multi-organ failure leading to increased fatality, is due to the consequence of poorly regulated inflammatory cytokine storm [11]. Finally, the inter-relationship between diabetes and COVID-19 should trigger more research to understand the extent to which specific mechanisms of the virus might contribute to worsening of glycaemic control and, in some cases, to the striking development of diabetic ketoacidosis or hyperglycaemic hyperosmolar syndrome, and possibly the development of new-onset diabetes.

People with comorbidities are especially vulnerable to the complications of COVID-19. There is recent surge of publications on COVID infection mostly from one region of China and hence generalization of the finding may not be possible because of paucity of data from other parts of the world. The present 
commentary focuses primarily on how to clinically examine and manage diabetes in the current COVID pandemic. It is expected that with availability of more credible data, the guidelines will be further refined and the management will become simpler. The risk for type 2 diabetes increases with age, and COVID-19 is more dangerous to people over 60. Aside from taking standard precautions to minimize exposure to the virus, the best thing your patients can do to manage their risk of COVID-19 is to continue to practice good diabetes management. Poorly controlled blood glucose contributes to immunosuppression and can increase risk [12].

\section{CONCLUSION}

Diabetes patients with COVID-19 infection are more prone for increased mortality, ICU admission, and prolonged hospital stay compared to their non-diabetic counterparts. COVID-19 with diabetes need strict glycemic control with insulin, and oral diabetic agents should be avoided when admitted to ICU. Diabetic patients should keep their blood sugar under control by doing frequent monitoring using digital appliance and to contact with their physician to remain in good glycemic control.

Overall the Indian climatic condition, good knowledge, traditional culture and food habit may reduce / slow down the spread of pandemic COVID-19 in India by which health care systems has sufficient time to prepare and assimilate the impact for better and healthy standards of living. Future research is urgently needed to provide a better understanding regarding potential differences in genetic predispositions across populations, underlying pathophysiological mechanisms of the association between COVID-19 and diabetes, and its clinical management.

\section{REFERENCES}

[1] Anjali gupta, AK Tripathi, RL Tripathi, SV Madhu and BD Benarjee. Advanced glycosylated end products-mediated activation of polymorphonuclear neutrophils in diabetes mellitus and associated oxidative stress. Indian J. Biochem. Biophys., Vol 44, October 2007, pp. 373-378.

[2] Park K. Epidemiology of chronic non-communicable diseases and condition in Park's Textbook of Preventive and Social Medicine. $20^{\text {th }}$ ed. Vol. 6. Jabalpur, India: Banarsidas Bhanot; 2009. p. 341.

[3] CE Mair et al., Antiviral and antiproliferative in vitro activities of piperamides from black pepper. Planta Med 2016; 82(S 01): S1S381. 
[4] Julio Cesar Escalona-Arranz et al., Antimicrobial activity of extracts from Tamarindus indica L. leaves. Pharmacogn Mag. 2010 Jul-Sep; 6(23): 242-247.

[5] Soheil Zorofchian Moghadamtousi et al., A Review on Antibacterial, Antiviral, and Antifungal Activity of Curcumin. Biomed Res Int. 2014; 2014: 186864.

[6] Carolyn R. Sturge, Felix Yarovinsky. Complex Immune Cell Interplay in the Gamma Interferon Response during Toxoplasma gondii Infection. Infect Immun. 2014 Aug; 82(8): 3090-3097.

[7] Atia sharif et al., A review on bioactive potential of benzoin resin. International Journal of Chemical and Biochemical Sciences, 10(2016): 106-110.

[8] Williamson E Walker AJ Bhaskaran $\mathrm{K}$ et al. Factors associated with COVID-19-related hospital death in the linked electronic health records of 17 million adult NHS patients. $J$ Chem Inf Model. 2019; 53: 16891699.

[9] Barron E Bakhai C Kar P et al. Associations of type 1 and type 2 diabetes with COVID-19-related mortality in England: a wholepopulation study. Lancet Diabetes
Endocrinol. 2020; (published online Aug 13.).

https://doi.org/10.1016/S2213$\underline{8587(20) 30272-2}$.

[10] Prabhakar Reddy E ; Manikandan S, Srikumar R; Vijayakumar R; Manoharan A; Naveen Kumar C; Ramesh S. Existing Knowledge on COVID-19 Pandemic and Hygienic Practice Among South Indians. Indian Journal of Public Health Research \& Development, November 2020, Vol. 11, No. 11.141-147.

[11] Bornstein SR, Dalan R, Hopkins D, Mingrone G, Boehm BO. Endocrine and metabolic link to coronavirus infection. Nat Rev Endocrinol 2020; 16: 297-8.

[12] Reddy EP et al., Role of Immunity: Current Status of COVID-19-A Review. British Journal of Medical and Health Research 2020. 